\title{
A Qualitative Exploration of Consultant Anaesthetists Attitudes to, and Experiences of, Perioperative Medication Errors in Emergency and Elective Theatre Settings
}

\author{
$N$ Wilson-Baig ${ }^{1,2^{*}}$ (D) and $R / s b a^{1,3}$ \\ ${ }^{1}$ Lancaster Medical School, Lancaster University, UK \\ ${ }^{2}$ Manchester University NHS Foundation Trust, UK \\ ${ }^{3}$ North Manchester General Hospital, UK
}

\begin{abstract}
Summary
In the UK, medication errors account for up to $20 \%$ of all reported adverse events in hospitalised patients. The potential for medication errors to occur during the peri-operative period is greater than in some other clinical settings and there is a paucity of good-quality evidence examining contributing factors.

A qualitative, interview-based approach was used, whereby consultant anaesthetists were asked about their experiences of peri-operative medication errors. Interview transcripts were thematically analysed to identify common themes describing factors that may contribute to peri-operative medication errors.

Ten consultant anaesthetists from a single hospital department in the North of England participated, with theoretical saturation being achieved. Major themes included human factors, organisational factors, non-technical skills, blame culture associated with error investigation, trust, and education. Suggested strategies to minimise errors included the use of technology and a collaborative approach between the organisation and anaesthetists in addressing human factors, error investigation, eliminating blame culture, enhancing non-technical skills, and education.
\end{abstract}

This study provides an insight into why peri-operative medication errors might occur, and what might be done to reduce them. Whilst some of the causes of errors, and strategies to reduce them, were in keeping with the literature, some new themes emerged or were reframed. This work suggests a collaborative and system-centred approach to addressing perioperative medication errors that involves the organisation and individuals.

\section{Keywords}

Peri-operative, Medication error, Medicine administration, Medication error prevention, Human factors, Organisation factors

\section{Introduction}

In the UK, medication errors (ME) account for $10 \%-20 \%$ of all reported adverse events in hospitalised patients [1]. The peri-operative period is of particular concern, as the potential for medication errors is greater than in some other clinical settings [1]. Despite this, the error rate during the peri-operative period remains unclear. Retrospective studies quote the error rate as between 1 in 133 and 1 in 274 anaesthetics given [2,3], whilst a prospective observational study reported 1 error in 20 anaesthetics given [4]. There is also a paucity of good-quality evidence examining factors that contribute to peri-operative medication error. Although published guidelines exist on how peri-operative medication errors can be prevented, they are based on studies with variable reliability and validity [4]. The available literature on peri-operative $\mathrm{ME}$ is based on self-reported data [2,3]. This data is either sourced from self-reported error reports or facilitated incident reporting [2-4].

An issue with many of these studies is that a definition of
*Corresponding author: Noamaan Wilson-Baig, BSc MSc (Clin Res), MSc (Clin Pharm), MBBS FRCA MRPharmS, ST7 NIHR ac- ademic specialty registrar in anaesthesia in the North West Deanery, honorary researcher, Lancaster Medical School, Lan- caster University, Lancaster, UK, E-mail: easinorm@me.com
Accepted: February 13, 2021
Published online: February 15, 2021
Citation: Wilson-Baig N, Isba R (2021) A Qualitative Exploration of Consultant Anaesthetists Attitudes to, and Experiences of, Perioperative Medication Errors in Emergency and Elective The- atre Settings. J Clin Anesth Pain Manag 5(1):198-204 
Citation: Wilson-Baig N, Isba R (2021) A Qualitative Exploration of Consultant Anaesthetists Attitudes to, and Experiences of, Perioperative Medication Errors in Emergency and Elective Theatre Settings. J Clin Anesth Pain Manag 5(1):198-204

peri-operative ME is not provided [5]. Although a definition of 'medication error' exists [6], it is a general description of medication error and does not acknowledge the difference in the way medicines are handled in the peri-operative period and on a general ward. Generally, on the wards, the medication process consists of transcribing the medicines a patient is taking on admission on to a prescription, prescribing new medicines, dispensing the medicines, double-checking medication with a colleague, and, finally administering the drug against a valid prescription [7]. This is usually done by individuals in a focussed manner where the individual is not disturbed unless it is an emergency.

The preparation and administration of medicines during the peri-operative period, especially in an emergency, differs from the general ward in that medicines are often prepared and administered by anaesthetists or a delegated member of the peri-operative team, whilst the team are concurrently performing multiple tasks where time may be of the essence, especially when managing a deteriorating patient [4]. Multitasking has itself been associated with ME [8]. Due to the nature of anaesthesia and managing emergencies, medicines are often administered without a written prescription. In such cases, once administered, the medicines are then transcribed onto the anaesthetic/prescription chart [4]. The peri-operative administration of medicines therefore presents a unique challenge in terms of patient safety.

Despite these differences, the common process is that the medicine has to be selected correctly by reading the label correctly, preparing the medicine as per the manufacturer's recommendation, storing the preparation in such a way that the medication would be easily accessible when required, and finally selecting the correct syringe prior to administration. Currie, et al. [9] found that 54\% of ME were attributed to the choice of the wrong drug vial.

There have been several published recommendations for reducing peri-operative ME [10-12]. These include the use of pre-filled syringes [13], bar-code technology [14,15], or use of distinctive packaging to differentiate medicines with similar names $[10,12]$. Other strategies include using guidelines to prompt the anaesthetist to carefully read the vial before preparation, ensure the clarity of labels, double-check preparations with a second colleague, and the use of standardised drug drawers and workspaces [11]. Incident reporting has also been suggested as a valuable tool in learning from adverse events as this can lead to improved patient safety [16].

The efficacy of these strategies has been evaluated in the literature. For example, 'TALL man lettering', where part of the medication name is printed in tall letters [17] is a system which uses uppercase letters to help differentiate medicines from others with similar names. TALLman lettering has been promoted by national organisations, including the U.S. Food and Drug Administration (FDA) [18]. A survey in 2008 showed $87 \%$ of respondents felt that the use of TALL man lettering had reduced errors in drug selection [17]. Pre-filled syringes could mitigate substitution error however, they still would have to be correctly selected from the medicine cabinet and tray prior to administration. Webster, et al. [2] found that
$29 \%$ of errors occurred due to a lack of a re-check prior to administration. Almghairbi, et al. [13], devised a 'rainbow tray' that utilised a colour-coding schema for medicines. This was found to be an effective and low-cost method for organising work trays containing medication. Most hospitals now use coloured trays to store and differentiate emergency medicines from medicines to be administered regionally, e.g. for nerve blocks.

Historically, double-checking has been a recommended strategy to reduce errors. Jensen, et al. [12] stated that double-checking was the single most effective measure with regards the prevention of ME. Double-checking is supported by the Department of Health [1] and is encouraged in other guidelines [11]. Conversely, in their study, O'Connell, et al. [19] found that nurses felt more confident using a single-check procedure as it gave them a sense of accountability for the medicine administration process. When two humans are checking a medicine, each individual is relying on the other to be more vigilant than they are in ensuring the correct medicine has been prepared [14]. The responsibility of the checking process therefore becomes diffused. Double-checking could inadvertently become a ritualistic process. Evley, et al. [14] describes this as involuntary automaticity whereby the cognitive process takes place unconsciously. This can defeat the purpose of double-checking. Evley, et al. [14] found the use of an electronic second-check mechanism, which produced an audible name of a bar coded syringe negated the need to have a second person to act as a double-checker. The disadvantages of this system included logistics, storage requirements, and costs of implementing and maintaining this system. Merry, et al. [15] demonstrated a 21\% reduction in ME when using a system that electronically recorded the administration of anaesthetic medicines. Despite recommendations for the use of barcode systems to identify medicines [20], few hospitals have taken up this technology. With regard to guidelines, most hospitals have their own medicines policy/guidelines, which covers handling of medicines. These are usually based on previous incidents or published literature $[11,12]$. However, they tend to be a generic list of processes as opposed to a means of empowering the practitioner to recognise and formulate plans to avoid situations with an error potential [21].

Incident reporting is considered to be an essential tool in improving patient safety $[11,16]$. However, only $10 \%$ of errors are reported voluntarily [16]. Suggested reasons for this include a belief that reporting incidents is not part of someone's job, a fear of disciplinary/legal action from the organisation, a fear that that the individual's competency will be brought into question, concerns about not being supported by the organisation or colleagues, and a dislike of the organisation's reporting system [16]. Organisational issues include unfamiliarity with the organisation's reporting system, the appropriateness of the reporting form, the time-consuming nature of incident reporting, a heavy workload, and a lack of satisfaction in the organisation's response to the incident, i.e. a feeling of nothing ever gets done [16].

Human factors are a topic of current interest in anaesthesia $[11,22]$ and have been identified to be an element in 
Citation: Wilson-Baig N, Isba R (2021) A Qualitative Exploration of Consultant Anaesthetists Attitudes to, and Experiences of, Perioperative Medication Errors in Emergency and Elective Theatre Settings. J Clin Anesth Pain Manag 5(1):198-204

peri-operative ME. The definition of human factors refers to a science that combines psychology and engineering, with the aim of enhancing performance whilst minimising harm [22]. Over time, 'human factors' has become synonymous with 'human failure', e.g. fatigue [22].

The purpose of this study was to explore the attitudes to, and experiences of, peri-operative medication errors from the perspective of anaesthetists-healthcare professionals who regularly and routinely handle medication during the peri-operative period.

\section{Methods}

Full prospective ethical approval was gained from the Faculty of Health and Medicine Research Ethics Committee (reference number FHMREC16140) of Lancaster University, and the Research and Development department at the Trust where the research was conducted.

This study used a qualitative approach, whereby the experiences of the research participants were explored. The aim was to identify themes as to why peri-operative ME occur, from the perspective of anaesthetists within their work setting. The data were generated via the use of semi-structured interviews carried out by the main author (NW-B).

This study took part in a single NHS Trust in the North of England. Consultant anaesthetists employed by the trust were invited to participate. At the time of the study, twenty consultant anaesthetists were working for the trust and were invited to participate.

Participants were recruited using an opportunistic, personal approach, supported by snowballing. Potential participants were provided with an information leaflet, an explanation of the study, and given two weeks to decide whether or not they would like to participate. All participants included in the study provided written consent. The minimum acceptable recruitment figure was ten. This was based on a similar study conducted on an intensive care unit [23]. The only exclusion criterion was working exclusively in maternity theatres. This is because medicines used in that setting are pre-packaged and prepped for use prior to an emergency. There is also a standard regime of medicines that are administered to women undergoing obstetric-related procedures with central neuraxial blockade being the anaesthesia of choice.

The questions used in the semi-structured interviews (Table 1) were based on existing literature where interviews were conducted in a hospital setting to identify contributory factors to ME $[24,25]$ and barriers to error reporting [26]. Although these studies were not conducted within the peri-operative setting, the interview questions used within these studies provided a basis for this study.

Data was collected by audio recordings of the interviews using an Olympus digital voice recorder. After each interview, the recording was transcribed into a word document and triple checked by the researcher against the recording. An alphanumeric code was assigned to each transcript to ensure anonymity, (e.g. 'ConsultantX/date (CX210617)') and the original recordings destroyed.

\section{Data analysis}

After each interview, each transcript was manually analysed using a process of open coding. Coding is a widely accepted method for analysing qualitative data [27]. Labels are applied to segments of the narrative with the aim of summarizing each piece of data from the interview transcript. The codes attempt to portray a concise meaning and explanation for responses given and provide a means interpretation of each piece of data. Theoretical explanations are then developed to explain what the piece of narrative might mean.

The data was analysed using Braun and Clarke's method of thematic analysis [28]. Themes were generated from the initial codes assigned to the data. Thematic analysis is a process for identifying and examining patterns within the data. It is considered theoretically flexible and can be applied to research questions like the one posed in this study [28].

Using this approach, each interview transcript was analysed, and descriptive codes applied to segments of data, for example, knowledge, fatigue, or stress. Themes were then

Table 1: Semi-structured interview questions used in the study.

\begin{tabular}{|l|l|}
\hline 1 & Can you tell me a bit about yourself? \\
\hline 2 & How long have you worked at the trust as a consultant anaesthetist? \\
\hline 3 & How long have you been practicing anaesthetics for? \\
\hline 4 & What does the term "peri-operative medication error" mean to you? \\
\hline 5 & Can you talk me through a time when you were involved in a peri-operative medication error? \\
\hline 6 & $\begin{array}{l}\text { In your experience is there a difference between how peri-operative medication errors occur between emergency and elective } \\
\text { theatres? }\end{array}$ \\
\hline 7 & What do you think are the contributory factors to peri-operative medication errors? \\
\hline 8 & What are your views on administering medication as a result of a verbal order? \\
\hline 9 & How about if someone hands you a syringe of a medicine to administer to a patient? \\
\hline 10 & What influences your decision to report a peri-operative medication error? \\
\hline 11 & What about near misses? \\
\hline 12 & What do you think we as a profession can do to reduce peri-operative medication errors? \\
\hline
\end{tabular}


Citation: Wilson-Baig N, Isba R (2021) A Qualitative Exploration of Consultant Anaesthetists Attitudes to, and Experiences of, Perioperative Medication Errors in Emergency and Elective Theatre Settings. J Clin Anesth Pain Manag 5(1):198-204

generated from these codes. Themes concisely describe multiple related codes by a unifying term. For example, the theme 'human factors' encompasses the codes, knowledge, fatigue and stress.

\section{Results}

Ten consultant anaesthetists participated $(50 \%$ of the total number of consultant anaesthetists working at the hospital). All participants worked full-time and the number of years worked as a consultant ranged between one and 17-years. Theoretical saturation was achieved around the main themes. After ten interviews, it was decided data collection would cease as all participants were constantly responding with similar answers. Additionally, there was a lack of interest from further anaesthetists in participating in the study.

When defining peri-operative medication error, participants described what they understood to be the definition of a ME and that this was something that occurs from before surgery to when the patient is discharged. One participant stated that they were not aware of a standard definition of $\mathrm{ME}$ in the peri-operative period. Although the definition of peri-operative period from participants was loosely in keeping with the definition provided by the Royal College of Anaesthetists [29], this being, 'the time from 24 hours before surgery until the point of discharge from hospital' [29], the definition of ME was not so simple. Most participants stated that an ME was related to either dispensing or administering the wrong medication or administering an overdose. This was partially in keeping with the definition provided by the Medicines and Healthcare products Regulatory Agency (MHRA). The MHRA define medication error as, 'an error in the process of prescribing, preparing, dispensing, administering, monitoring or providing advice on medicines' [6].

All participants were able to provide anecdotes of personal experiences of ME. They were able to recount what happened, how they managed it, and what learning points were gleaned from the incident. The participants also shared what they thought were the contributory factors in the context of the incident they were involved with and also generally within anaesthetic practice. All types of error were in keeping with published literature [2-4]. Substitution (40\%), overdose (40\%) and dosing errors (20\%) were described by participants. Although participants appreciated that ME are inevitable throughout the career of an anaesthetist, all participants described the challenges of error reporting and how the employing organisation should adopt a collaborative approach when investigating ME. Finally, the participants provided suggestions on how ME could be mitigated in the peri-operative period.

The main thematic categories which emerged from this study included non-technical skills (reading the label, selecting the right medication, double-checking process), organisational factors (staffing, rotas, equipment availability, error reporting systems, error investigation), human factors (fatigue, vigilance, distraction, communication, trust), and education. When considering error reporting, the recurring themes were blame culture, reporting process, organisational factors, and education. Owing to the large crossover in themes, the re- sults have been grouped into three categories: contributory factors, error reporting, and error prevention.

\section{Contributory factors}

All ten participants attributed both organisational factors and human factors as contributory to ME. There was a feeling that organisations did not appreciate human factors. This was captured by one participant:

...human factors in an emergency situation plays a big part of the outcome. I don't think the trust acknowledges this when they do investigations...

Fatigue was also reported by all participants as a contributing factor. This was in the context of organisational issues and captured by another participant thus:

Fatigue is very important. So, breaks, adhering to the European working time directive, making sure the shifts are not long with breaks in between... and that is something and that needs to be addressed here. Again, with the economic pressures and staffing issues.

\section{Error reporting}

All participants felt all errors should be reported, especially if a patient was harmed. They felt that lessons could be learnt through investigation and dissemination of MEs. Organisations should take responsibility, consider the whole incident, and ensure appropriate guidelines were in place. One participant said:

We need to follow it up with a root cause analysis and once the root cause analysis is done, we need to make some stronger valid recommendations and definitely follow these. The root cause analysis must also consider the whole scenario to make sure a robust guideline is in place.

However, participants also found the reporting process challenging, and noted that terminology within forms could appear accusatory.

It's so complex now and you can't actually pick the phrase you want as you have to use their phrases, so you have an instigator and a victim. These phrases tend to be accusative terms and may not be reflective of the situation.

\section{Error prevention}

All participants felt that addressing human factors, non-technical skills, including reading and selecting the right medicine, organisational issues, and education were integral to error prevention.

Various strategies anaesthetists could employ to reduce medication errors were suggested.

When you are loading any medications, you have a second person if possible, to double check.

We need to introduce technology to make sure drugs can be scanned on an electronic database system.

In an emergency situation I think we should have prefilled syringes, and this would avoid having to worry about it because it comes prefilled and it says what it is. 
Citation: Wilson-Baig N, Isba R (2021) A Qualitative Exploration of Consultant Anaesthetists Attitudes to, and Experiences of, Perioperative Medication Errors in Emergency and Elective Theatre Settings. J Clin Anesth Pain Manag 5(1):198-204

I deliberately use different size syringes for different medications. If I have to draw up morphine, I will always draw up in $10 \mathrm{~mL}$ syringe.

There are the usual guidelines for good practice like having to check that the right drug has been selected, drawn up correctly and checked against the drug chart... as an anaesthetist I have to make sure is it the right drug and has the right label.

Trust was a factor in error prevention, with anaesthetists preferring to prepare their own medicines. However, if they worked with trusted colleagues, e.g. the Operation Department Practitioner (ODP), or an experienced trainee, then this would be less of an issue. One anaesthetist said:

It will depend on how much I trust my colleague. If I trust them a lot and I don't know of any probity issue of my colleague, then probably I wouldn't be that unhappy to give that medication to the patient.

All participants felt education for all healthcare professionals without a no-blame culture is essential in mitigating errors. Examples from two participants were,

The key thing is education and having an open no blame culture, because everybody is worried somebody will blame me for something.

Not only for doctors but for nurses as well, adequate training, adequate knowledge, adequate experience is mandatory and there should be some standard operating procedures guidelines protocols in the background.

\section{Discussion}

This study was designed to explore medication errors from the perspective of consultant anaesthetists who handle medicines during the peri-operative period. Whilst some of the causes of errors described by participants and the strategies for reducing them were in keeping with the literature, some new themes emerged or were reframed.

Defining peri-operative ME was challenging for participants. This is not wholly unexpected as, at the time of writing, there is not a single, standardised, accepted definition of peri-operative ME. Historically the definition of medication error has not been straightforward. In their systematic review, Lisby, et al. [5] retrieved 26 definitions of 'medication error'. Although this has been addressed by the MHRA [6], the definition of 'medication error' provided by the MHRA does not acknowledge the difference between the way medicines are prepared in a dispensary/ward and that during the peri-operative period.

Human factors and organisational issues were felt to be the main contributory factors to peri-operative ME. Fatigue was the most reported theme in this study. The Health Safety Executive (HSE) [30] define fatigue as, "a result of prolonged mental or physical exertion", which, "can impair performance and mental alertness". In 2006, the HSE introduced a fatigue index for use in the manufacturing industry and suggested that this index could be used in healthcare [30]. The index considers the cumulative effects of fatigue, the workload, job type, length of breaks, and, length of time on duty. Howev- er, the causes and effects of fatigue vary between individuals and although an index can provide some information, it is challenging to objectively assess fatigue [31]. The roles that healthcare professions do is very different to that of the manufacturing industry. For example, someone working for a car company will have predefined targets with set tools to make a vehicle of a certain specification. Healthcare professionals on the other hand have to respond and attempt to manage deteriorating patients with multiple comorbidities and varying age groups. Unlike the car industry, the peri-operative team are managing highly complex and unpredictable situations with multiple unknowns and therefore potentially multiple unknown failure possibilities. Healthcare professionals may also not be aware that they are fatigued and so may continue to work due to the urgent nature of the job. Participants felt that organisations have a role in ensuring fatigue is addressed by employing adequate staff, encouraging adequate breaks, and providing adequate and fully functional equipment. The HSE has acknowledged that in the manufacturing industry, the effect of fatigue for permanent day shift workers is different from those who work permanent night shifts. The challenge in healthcare is that people work both night and day shifts with a short turn around and not much is known about the effect of fatigue in these individuals. Recently, the Association of Anaesthetists have recently published a fatigue tool with resources to assist anaesthetists to assess for themselves whether they might be suffering from fatigue and what they can do to manage fatigue [32]. Further research is required on how this work pattern impacts on fatigue.

All anaesthetists appreciated the importance of incident reporting of ME. However, the logistical hurdle of the reporting process was felt to be a hinderance. The computer system was protracted and not always easy to navigate. There was a feeling, particularly from experienced anaesthetists, that recording errors in a dedicated notebook, was user friendly, and there was a freedom to note down the context of the error in the practitioner's own words. Computers were not always accessible, and the rigours of the working day were not conducive to completing incident forms. Participants also felt that the generic nature of the form did not allow the individual to record the complexities of the case from the perspective of the anaesthetist, or what the thought processes were of the individuals concerned. Only facts relating to the incident were recorded. Anaesthetists felt that any subsequent investigation could not truly know what happened as the context of what occurred was not recorded. As a result, investigations can unintentionally succumb to hindsight bias [33]. Inadvertently the complex nature of the episode becomes structured and simplified [34] as the information provided on the incident form is related to a known outcome by the investigating team. In an attempt by the organisation to provide an explanation of what happened, counterfactuals are generated. These are based on guidelines or professional standards, and an assessment is made as to whether a deviation was made from the guidelines. Unfortunately, this approach can result in judging individuals for not adhering to the guidelines [33]. This could be an explanation of why 'blame culture' still pervades within healthcare and why some people may be reluctant to complete incident forms as the individual would 
Citation: Wilson-Baig N, Isba R (2021) A Qualitative Exploration of Consultant Anaesthetists Attitudes to, and Experiences of, Perioperative Medication Errors in Emergency and Elective Theatre Settings. J Clin Anesth Pain Manag 5(1):198-204

not want to risk disciplinary taken action against them taken for something, they believe was not their fault. Blame culture was a major theme in this study. Although participants appreciated that organisations were attempting to eliminate blame culture, they felt blame culture still exists. Based on the responses from this study, the incident reporting process should be reviewed with the aim of having a system that is quick to complete, simple to use and allow the individual to provide a narrative of what happened in real time and in the anaesthetist's own words. The investigation process should also be reviewed. The aim of any incident analysis is to; firstly, reconstruct the sequence of events of what happened in real time with consideration for the organisational environment within which the error occurred, and secondly, recreate the mindset of the individuals present whilst acknowledging that decisions made by these individuals were being made in an evolving situation where the outcome, the error, was unknown until it happened $[33,34]$.

When considering strategies for error prevention, various suggestions were made. For example, the use of double-checking, technology, pre-filled syringes, or coloured trays to store medicines for use once prepared. However, common to any strategy will be that the anaesthetist has to select the correct vial by correctly reading the label, prepare the medication as per the manufacturer's recommendation, apply a correct label to the preparation, and finally store the preparation in such a way that the medicine would be easily accessible when required. Participants commented on the challenge of having to quickly select the correct medicine from a 'bunch of syringes' in a tray, especially in an emergency. Anaesthetists spoke about using their own in-built selfcheck processes that serve as a personal second check. These processes, or rituals are developed during the training years of the consultant, act as a personal supplement to published guidelines or professional standards. For example, most anaesthetists will only use $2 \mathrm{~mL}$ syringes when preparing short acting opioids. The syringe size acts as a 'soft check' that the medicine in the syringe is an opioid. However, confusion can arise when the muscle relaxant suxemethonium is also drawn up in a $2 \mathrm{~mL}$ syringe. Aware of the risk of $\mathrm{ME}$, consultant anaesthetists are keen to teach trainees their methods of avoiding errors, especially those trainees who have not worked with them before. To the consultant, theirs is a tried and tested method of avoiding errors. To the trainee, they have to learn the foibles of each consultant anaesthetist they work with whilst developing their own self-check processes. Organisational guidelines were seen to be a beneficial tool to mitigating ME. However, guidelines were also seen as a generic list of processes as opposed to a means of empowering the practitioner to recognise and formulate plans to avoid situations with error potential [21]. Guidelines do not cater for every situation and complex situations are oversimplified into a dichotomous bifurcation that is a choice between a wrong decision and a correct one.

All participants felt organisations should take more responsibility and encourage a supportive environment when preventing ME. Education was seen as something that organisations and governing bodies could use to mitigate errors.
Marshall and Chrimes [11] published a twelve-point plan for anaesthetists and recommended that the handling of medication should be essential training for junior trainees entering the anaesthesia programme. This was echoed by participants in this study. Medicine handling is an integral component of undergraduate programs like Pharmacy but not in Medicine. Considering the harm that ME can cause, could medicine handling be considered for inclusion in the curriculum at medical school?

The qualitative methodological approach used in this study presented some limitations. This could be deemed as subjective, and results influenced by the researcher's own beliefs. Sadler [35] described how a researcher's prior knowledge, experience, emotional background or outlook could be a source of bias in qualitative research, and that the interpretation of results could be influenced by the personal preferences of the researcher. Multiple researchers could mitigate this by bringing in a new perspective to the coding process thereby strengthening the validity and reliability of the study. The primary researcher in this study (NW-B) has ten years' experience working as a pharmacist, where dispensing medicines are an essential component of the role. This could contribute to researcher bias. In order to address this, reflexivity was applied to the study by the author. Reflexivity encompasses the influence researchers can have on qualitative research [36] and is a valid process of self-reflection that increases the rigour of qualitative research [37].

A strength of this study is that it provided a valuable insight into the attitudes and experiences of peri-operative ME from the perspective of those who directly handle medicines in the acute setting, and the focus of the work was depth of understanding rather than breadth. Although other studies have adopted a qualitative approach, this is the first that addresses the whole issue of peri-operative ME as opposed to specific aspects, e.g. the use of a rainbow tray, or an electronic barcode system to mitigate errors $[13,14]$.

A study weakness was that it may lack broad generalisability. The study was conducted at a District General Hospital, which has different challenges logistically, clinically, managerially, and from a human resource perspective to a large teaching hospital. Larger hospitals employ larger numbers of anaesthetists, deal with complex cases, and have a larger workload and so potentially there is a larger room for errors [2]. It was also challenging to conduct interviews as many times these were cancelled/rescheduled, or the anaesthetist was on leave. Another weakness is that the study was conducted by a single researcher. The study would have been strengthened through an independent review process for adequacy and validity.

Future research could focus on emergent themes from this study. For example, the relationship between the organisation, human factors, the anaesthetist, trust, incident reporting, and blame culture. The process of incident reporting needs addressing-instead of relating actions to a known outcome (the error), any investigation should focus on identifying the narrative of what happened. This would result in a better understanding of the incident. Additionally, the useful- 
Citation: Wilson-Baig N, Isba R (2021) A Qualitative Exploration of Consultant Anaesthetists Attitudes to, and Experiences of, Perioperative Medication Errors in Emergency and Elective Theatre Settings. J Clin Anesth Pain Manag 5(1):198-204

ness of guidelines in ME prevention is not clear and does not take into consideration goal conflicts that happen especially in emergency situations.

This study provided a useful in-depth insight into why peri-operative medication errors might occur and what could be done to reduce them. This work suggests a collaborative and system-centred approach to addressing MEs that involves the organisation and individuals on the front-line.

\section{Acknowledgement}

I would like to acknowledge Professor Andrew Smith for kindly advising on the preparation of this manuscript.

\section{Competing Interest}

This study was funded by the National Institute for Health Research Academic Clinical Fellow (NIHR ACF) Programme. Award Number ACF-2015-05-001.

\section{References}

1. Department of Health (2004) Building a safer NHS for patients: Improving medication safety. London, 84-89.

2. Webster CS, Merry AF, Larsson L, et al. (2001) The frequency and nature of drug administration error during anaesthesia. Anaesth Intensive Care 29: 494-500.

3. Llewellyn RL, Gordon PC, Wheatcroft D, et al. (2009) Drug administration errors: A prospective study survey from three South African teaching hospitals. Anaesth Intensive Care 37: 93-98.

4. Nanji KC, Patel A, Shaikh S, et al. (2016) Evaluation of perioperative medication errors and adverse drug events. Anesthesiology 124: 25-34.

5. Lisby M, Nielsen LP, Brock B, et al. (2010) How are medication errors defined? A systematic literature review of definitions and characteristics. Int J Qual Health Care 22: 507-518.

6. MHRA, NHS England (2014) Improving medication error and incident reporting and learning.

7. Nadzam D (1998) A systems approach to medication to medication use. In: Cousins DD, Medication use: A systems approach to reducing errors. (edn), Joint Commission on Accreditation of Healthcare Organizations, Oakbrook Terrace, IL, 5-18.

8. Reason J (2000) Human error: Models and management. BMJ 320: 768-770.

9. Currie M, Mackay P, Morgan C, et al. (1993) The Australian incident monitoring study. The wrong drug problem in anaesthesia: An analysis of 2000 incident reports. Anaesth Intensive Care 21: 596-601.

10. Association of perioperative Registered Nurses (2006) AORN guidance statement: Safe medication practices in perioperative settings across the life span. AORN J 84: 276-283.

11. Marshall SD, Chrimes N (2019) Medication handling: Towards a practical, human-centred approach. Anaesthesia 74: 280-284.

12. Jensen LS, Merry AF, Webster CS, et al. (2004) Evidence-based strategies for preventing drug administration errors during anaesthesia. Anaesthesia 59: 493-504.

13. Almghairbi DS, Sharp L, Griffiths R, et al. (2018) An observational feasibility study of a new anaesthesia drug storage tray. Anaesthesia 73: 356-364.

14. Evley R, Russell J, Mathew D, et al. (2010) Confirming the drugs administered during anaesthesia: A feasibility study in the pilot National Health Service sites, UK. Br J Anaesth 105: 289-296.

15. Merry AF, Webster CS, Hannam J, et al. (2011) Multimodal sys- tem designed to reduce errors in recording and administration of drugs in anaesthesia: Prospective randomised clinical evaluation. BMJ 343: d5543.

16. Pfeiffer Y, Manser T, Wehner T (2010) Conceptualising barriers to incident reporting: A psychological framework. Qual Saf Health Care 19: e60.

17. Grissinger M (2012) Tall man letters are gaining wide acceptance. PT 37: 132-148.

18. FDA Name Differentiation Project (2017) Center for drug evaluation and research.

19. O'Connell B, Crawford S, Tull A, et al. (2007) Nurses attitudes to single checking medications: Before and after its use. International Journal of Nursing Practice 13: 377-382.

20. Eichhorn JH (2010) Medication safety in the operating room: Time for a new paradigm. APSF Summit Conference Proceedings. Anaesthesia Patient Safety Foundation Newsletter 25: 1-20.

21. Reason J (2004) Beyond the organisational accident: The need for "error wisdom" on the frontline. Qual Saf Health Care 13: 28-33.

22. Russ AL, Fairbanks RJ, Karsh B, et al. (2013) The science of human factors: Separating fact from fiction. BMJ Qual Saf 22: 802-808.

23. Sanghera IS, Franklin BD, Dhillon S (2007) The attitudes and beliefs of healthcare professionals on the causes and reporting of medication errors in a UK intensive care unit. Anaesthesia 62: 53-61.

24. Armitage G, Newell R, Wright J (2010) Improving the quality of drug error reporting. Journal of Evaluation in Clinical Practice 16: 1189-1197.

25. Nichols $P$, Copeland T, Craib IA, et al. (2008) Learning from error: Identifying contributory causes of medication errors in an Australian hospital. The Medical journal of Australia 188: 276-279.

26. Hartnell N, MacKinnon N, Sketris I, et al. (2012) Identifying, understanding and overcoming barriers to medication error reporting in hospitals: A focus group study. BMJ Qual Saf 21: 361-368.

27. Charmaz K (2006) Constructing grounded theory: A practical guide through qualitative analysis. SAGE Publications, London.

28. Braun V, Clarke V (2006) Using thematic analysis in psychology. Qualitative Research in Psychology 3: 77-101.

29. The Royal College of Anaesthetists (2014) Perioperative medicine. The pathway to better surgical care.

30. Health and Safety Executive (2006) The development of a fatigue/risk index for shiftworkers.

31. Sadeghniiat HK, Yazdi Z (2015) Fatigue management in the workplace. Ind Psychiatry J 24: 12-17.

32. Association of Anaesthetists nd., Fatigue resources. [Accessed 20/10/2020].

33. Dekker SW (2002) Reconstructing human contributions to accidents: The new view on error and performance. J Safety Res 33: 371-385.

34. Reason J (1990) The contribution of latent human failures to the breakdown of complex systems. Philos Trans R Soc Lond B Biol Sci 327: 475-484.

35. Sadler DR (2002) Intuitive data processing as a potential source of bias in naturalistic evaluations. In: Huberman A, Miles MB, The qualitative researcher's companion. (edn), Sage Publications, Thousand Oaks, CA, 123-136.

36. Green J, Thorogood N (2009) Qualitative methods for health research. ( $\left.2^{\text {nd }} \mathrm{edn}\right)$, Sage Publications, London, 191-197.

37. Jootun D, McGhee G, Marland GR (2009) Reflexivity: Promoting rigour in qualitative research. Nurs Stand 23: 42-46. 